TRANSACTIONS OF THE

AMERICAN MATHEMATICAL SOCIETY

Volume 359, Number 8, August 2007, Pages 3669-3685

S 0002-9947(07)04311-5

Article electronically published on February 23, 2007

\title{
AN EXPLICIT FORM FOR KEROV'S CHARACTER POLYNOMIALS
}

\author{
I. P. GOULDEN AND A. RATTAN
}

\begin{abstract}
Kerov considered the normalized characters of irreducible representations of the symmetric group, evaluated on a cycle, as a polynomial in free cumulants. Biane has proved that this polynomial has integer coefficients, and made various conjectures. Recently, Śniady has proved Biane's conjectured explicit form for the first family of nontrivial terms in this polynomial. In this paper, we give an explicit expression for all terms in Kerov's character polynomials. Our method is through Lagrange inversion.
\end{abstract}

\section{INTRODUCTION}

1.1. Background and notation. A partition is a weakly ordered list of positive integers $\lambda=\lambda_{1} \lambda_{2} \ldots \lambda_{k}$ where $\lambda_{1} \geq \lambda_{2} \geq \cdots \geq \lambda_{k}$. The integers $\lambda_{1}, \ldots, \lambda_{k}$ are called the parts of the partition $\lambda$, and we denote the number of parts by $l(\lambda)=k$. If $\lambda_{1}+\cdots+\lambda_{k}=d$, then $\lambda$ is a partition of $d$, and we write $\lambda \vdash d$. We denote by $\mathcal{P}$ the set of all partitions, including the single partition of 0 (which has no parts). For partitions $\omega, \lambda \vdash n$ let $\chi_{\omega}(\lambda)$ be the character of the irreducible representation of the symmetric group $\mathfrak{S}_{n}$ indexed by $\omega$, and evaluated on the conjugacy class $\mathcal{C}_{\lambda}$ of $\mathfrak{S}_{n}$, which consists of all permutations whose disjoint cycle lengths are specified by the parts of $\lambda$.

Various scalings of irreducible symmetric group characters have been considered in the recent literature. The central character is given by

$$
\tilde{\chi}_{\omega}(\lambda)=\left|\mathcal{C}_{\lambda}\right| \frac{\chi_{\omega}(\lambda)}{\chi_{\omega}\left(1^{n}\right)}
$$

where $\chi_{\omega}\left(1^{n}\right)$ is the degree of the irreducible representation indexed by $\omega$. For results about the central character, see, for example, 4, 5, 8. Related to this scaling, for the conjugacy class $\mathcal{C}_{k 1^{n-k}}$ only, is the normalized character, given by

$$
\widehat{\chi}_{\omega}\left(k 1^{n-k}\right)=n(n-1) \cdots(n-k+1) \frac{\chi_{\omega}\left(k 1^{n-k}\right)}{\chi_{\omega}\left(1^{n}\right)}=k \widetilde{\chi}_{\omega}\left(k 1^{n-k}\right) .
$$

The subject of this paper is a particular polynomial expression for the normalized character. The statement of this expression requires some notation involving the partition $\omega$ of $n$. We adapt the following description from Biane [1, 2]: consider the Young diagram of $\omega$, in the French convention (see [10, footnote page 2]), and

Received by the editors April 20, 2005.

2000 Mathematics Subject Classification. Primary 05E10; Secondary 05A15, 20 C30.

(C)2007 American Mathematical Society 
translate it, if necessary, so that the bottom left of the diagram is placed at the origin of an $(x, y)$ plane. Finally, rotate the diagram counterclockwise by $45^{\circ}$ (this is often referred to as "Russian notation"). Note that $\omega$ is uniquely determined by

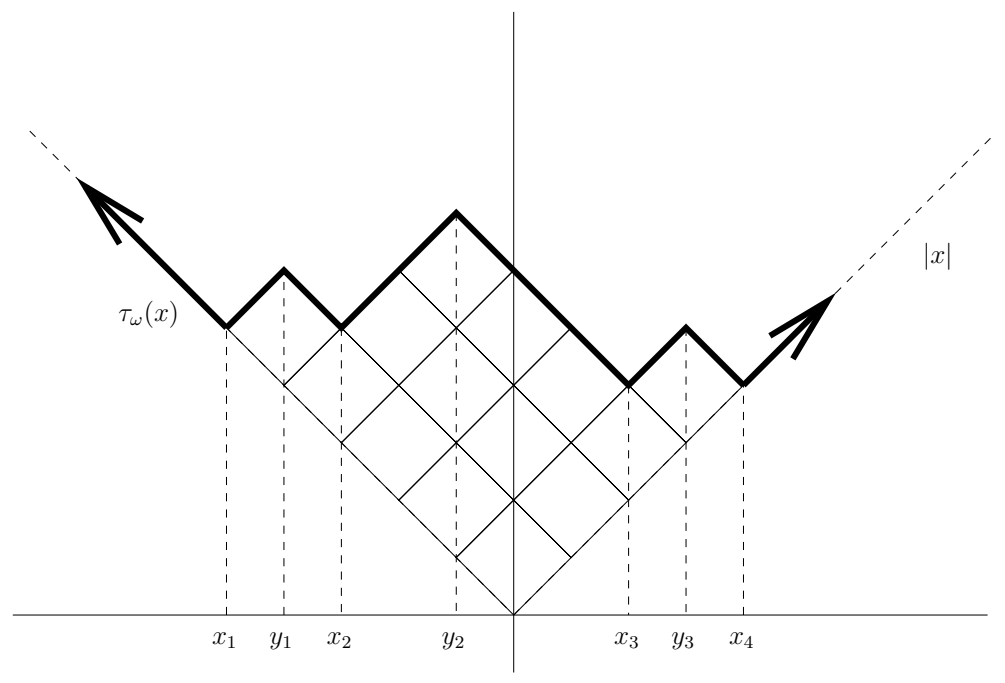

Figure 1. The partition (43331) of 14, drawn in the French convention, and rotated by $45^{\circ}$.

the curve $\tau_{\omega}(x)$ (see Figure 1). The value of $\tau_{\omega}(x)$ is equal to $|x|$ for large negative or positive values of $x$, and it is clear that $\tau_{\omega}^{\prime}(x)= \pm 1$, where differentiable. The points $x_{i}$ and $y_{i}$ are the $x$-coordinates of the local minima and maxima, respectively, of the curve $\tau_{\omega}(x)$. We suitably scale the size of the boxes in our Young diagram so that the points $x_{i}$ and $y_{i}$ are integers. Setting $\sigma_{\omega}(x)=\left(\tau_{\omega}(x)-|x|\right) / 2$, consider the function

$$
G_{\omega}(z)=\frac{1}{z} \exp \int_{\mathbb{R}} \frac{1}{z-x} \sigma_{\omega}^{\prime}(x) d x .
$$

Carrying out the above integration one obtains

$$
G_{\omega}(z)=\frac{\prod_{i=1}^{m-1}\left(z-y_{i}\right)}{\prod_{i=1}^{m}\left(z-x_{i}\right)}
$$

where $m$ is the number of nonempty rows in the Young diagram of $\omega$. Now let $R_{i}(\omega), i \geq 1$, be defined by

$$
z^{-1}+\sum_{i \geq 1} R_{i}(\omega) z^{i-1}=G_{\omega}^{\langle-1\rangle}(z)
$$

where $\langle-1\rangle$ denotes compositional inverse. Briefly, the origins of the series $G_{\omega}(z)$, and in fact Kerov's polynomials, come from attempting to answer asymptotic questions about the characters of the symmetric group. The generating series $G_{\omega}(z)$ is 
known as the moment generating series and is the series

$$
\int_{\mathbb{R}} \frac{1}{z-x} d\left(m_{\omega}\right)
$$

where $m_{\omega}$ is known as the transition measure of the diagram $\omega$. The measure is given by

$$
m_{\omega}=\sum_{k=1}^{m} \mu_{k} \delta_{x_{k}}
$$

where $\delta_{x_{k}}$ is the usual delta function and

$$
\mu_{k}=\frac{\prod_{i=1}^{m-1}\left(x_{k}-y_{i}\right)}{\prod_{i=1, i \neq k}^{m}\left(x_{k}-x_{i}\right)} .
$$

Thus, the coefficient of $(1 / z)^{k}$ in $G_{\omega}(z)$ is the $k^{\text {th }}$ moment of the measure $m_{\omega}$. In this context, the $R_{i}(\omega)$ 's are known as free cumulants of the measure $m_{\omega}$ in free probability theory (see Biane [1, Section 1.2 and Section 3]). Biane uses the theory of free probability to give asymptotic information about the characters of the symmetric group. More specifically, if $\sigma_{n} \in \mathfrak{S}_{n}, n \geq 1$, is a sequence of permutations (subject to a certain "balanced" restriction on the associated Young diagram) with $k_{i}$ cycles of length $i$ for $i \geq 2$ and $r=\sum_{i} i k_{i}$, then we have

$$
\frac{\chi_{\omega}\left(\sigma_{n}\right)}{\chi_{\omega}\left(1^{n}\right)}=\prod_{i \geq 2} R_{i+1}^{k_{i}}(\omega) n^{-r}+O\left(n^{-\frac{r+1}{2}}\right) .
$$

For more information about the asymptotics of characters of the symmetric group (and free cumulants) see, for example, [1, 7, 9].

1.2. Kerov's character polynomials. The particular polynomials that are the subject of this paper involve the $R_{i}(\omega)$ 's. They first appeared in Biane [2], where the following result is stated (as Theorem 5.1).

Theorem 1.1. For $k \geq 1$, there exist universal polynomials $\Sigma_{k}$, with integer coefficients, such that

$$
\widehat{\chi}_{\omega}\left(k 1^{n-k}\right)=\Sigma_{k}\left(R_{2}(\omega), R_{3}(\omega), \ldots, R_{k+1}(\omega)\right),
$$

for all $\omega \vdash n$ with $n \geq k$.

Biane attributes Theorem 1.1 to Kerov, who described this result in a talk at an IHP conference in 2000, but a proof first appeared in a later paper of Biane 3. The polynomials $\Sigma_{k}$ are known as Kerov's character polynomials. They are referred to as "universal polynomials" in Theorem 1.1 to emphasize that they are independent of $\omega$ and $n$, subject only to $n \geq k$. Thus we write them with $R_{i}(\omega)$ replaced by an indeterminate $R_{i}, i \geq 2$. In indeterminates $R_{i}$, the first six of Kerov's character polynomials, as listed in [2], are given below:

$$
\begin{aligned}
& \Sigma_{1}=R_{2}, \\
& \Sigma_{2}=R_{3}, \\
& \Sigma_{3}=R_{4}+R_{2}, \\
& \Sigma_{4}=R_{5}+5 R_{3}, \\
& \Sigma_{5}=R_{6}+15 R_{4}+5 R_{2}^{2}+8 R_{2}, \\
& \Sigma_{6}=R_{7}+35 R_{5}+35 R_{3} R_{2}+84 R_{3} .
\end{aligned}
$$


Note that all coefficients appearing in this list are positive. It is conjectured that this holds in general: that for any $k \geq 1$, all nonzero coefficients in $\Sigma_{k}$ are positive.

In Biane 2], this conjecture, which we shall refer to as the R-positivity conjecture, is attributed to Kerov. In fact, Biane [1, Section 7] has a conjectured combinatorial interpretation for the coefficients of the $R$ 's in Kerov's polynomials pertaining to Cayley graphs. The conjecture there is vague, however, and has been made more precise by Śniady 11 but is still far from being complete. Numerically, the Rpositivity conjecture has been verified for $k$ up to 15 by Biane [3], who computed $\Sigma_{k}$ for $k \leq 15$, using an implicit formula for $\Sigma_{k}$ [1, Theorem 5.1] that he credits to Okounkov (private communication). Biane further comments that "It seems plausible that S. Kerov was aware of this (see especially the account of Kerov's central limit theorem in 7)." The following result gives an adaptation of Biane's formula that appears in Stanley [12.

Theorem 1.2. Let $R(x)=1+\sum_{i \geq 2} R_{i} x^{i}$ and

$$
F(x)=\frac{x}{R(x)}, \quad H(x)=\frac{1}{F^{\langle-1\rangle}\left(x^{-1}\right)} .
$$

Then, for $k \geq 1$,

$$
\Sigma_{k}=-\frac{1}{k}\left[x^{-1}\right]_{\infty} \prod_{j=0}^{k-1} H(x-j) .
$$

Theorem 1.2 implicitly determines $\Sigma_{k}$ as a polynomial in the $R_{i}$ 's. For explicit formulas, it is convenient to consider separately the graded pieces of $\Sigma_{k}$, defined as follows: let the weight of the monomial $R_{j_{1}} \cdots R_{j_{i}}$ be $j_{1}+\cdots+j_{i}$. For $n \geq 0$, we define

$$
\Sigma_{k, 2 n}=\left[u^{k+1-2 n}\right] \Sigma_{k}\left(R_{2} u^{2}, \ldots, R_{k+1} u^{k+1}\right),
$$

the sum of all terms of weight $k+1-2 n$ in $\Sigma_{k}$. (From elementary parity considerations, all other coefficients in $\Sigma_{k}$ are 0 .) It is immediate that $\Sigma_{k, 0}=R_{k+1}$. An explicit formula is known for $\Sigma_{k, 2}$, and for the statement of this formula, we introduce polynomials $C_{m}$ in the $R_{i}$ 's, where $C_{0}=1, C_{1}=0$, and

$$
C_{m}=\sum_{\substack{j_{2}, j_{3}, \ldots \geq 0 \\ 2 j_{2}+3 j_{3}+\cdots=m}}\left(j_{2}+j_{3}+\cdots\right) ! \prod_{i \geq 2} \frac{\left((i-1) R_{i}\right)^{j_{i}}}{j_{i} !}, \quad m \geq 2 .
$$

The following explicit formula for $\Sigma_{k, 2}$ was conjectured by Biane [3, Conjecture 6.4], and proved by Śniady [11, Theorem 22]. Śniady's proof was obtained by finding and then solving an equivalent combinatorial problem.

Theorem 1.3. For $k \geq 1$,

$$
\Sigma_{k, 2}=\frac{1}{24}(k-1) k(k+1) C_{k-1} .
$$

Note that the R-positivity of $\Sigma_{k, 2}$ follows immediately from Theorem 1.3 , using (1.5).

For $n \geq 2$, only one explicit result is known, given in the following result for the linear coefficient, due to Biane [3] and Stanley [12].

Theorem 1.4. For $n \geq 1, k \geq 2 n-1$, the coefficient of $R_{k+1-2 n}$ in $\Sigma_{k, 2 n}$ is equal to the number of $k$-cycles $c$ in $\mathfrak{S}_{k}$ such that $(1 \ldots k) c$ has $k-2 n$ cycles. 
Finally, for higher order terms when $n=2$, the following conjecture of Stanley (private communication) has been communicated to us by Biane.

Conjecture 1.5. For $i \geq 1$,

$$
\left[R_{2}^{i}\right] \Sigma_{2 i+3,4}=\frac{1}{540} i(i+1)^{3}(i+2)^{3}(i+3)(2 i+3) .
$$

1.3. Outline of the paper. In this paper, we obtain an explicit formula for $\Sigma_{k, 2 n}$, where $k$ and $n$ are arbitrary. This is our main result, stated in Section 2 as Theorem 2.1. Variants are also given as Theorems 2.2 and 2.3. These results are a natural generalization of Theorem 1.3. since they give $\Sigma_{k, 2 n}$ as a polynomial in the $C_{m}$ 's, with coefficients that are rational polynomials in $k$. We call such an expression a $C$-expansion for $\Sigma_{k, 2 n}$. Based on significant amounts of data, we conjecture that $\Sigma_{k, 2 n}$ is $C$-positive (all nonzero coefficients are positive) for all $n \geq 1$ as Conjecture 2.4. This C-positivity conjecture is stronger than the R-positivity conjecture, immediately from (1.5).

In Section 3, we consider the special cases of our main result for $n=1$ and $n=2$. For $n=1$, this gives another proof of Theorem 1.3. For $n=2$, the expression for $\Sigma_{k, 4}$ that we obtain, in Theorem 3.3 , is new. We are able to specialize this expression to prove Conjecture 1.5. Also, we are able to prove the C-positivity conjecture for $\Sigma_{k, 4}$ as Corollary 3.5. Finally, we consider the linear terms in the $R_{i}$ 's, for arbitrary $n$, and obtain another proof of Theorem 1.4 .

In general, for $n \geq 3$, we are not able to prove the R-positivity conjecture or the C-positivity conjecture, perhaps because our methods are not combinatorial. Instead we apply the Lagrange inversion to "unwind" the compositional inverse in Theorem 1.2. This is carried out in Section 4, where we give the proof of the main result and variants.

\section{THE MAIN RESULT}

For the partition $\lambda \vdash n$ we denote the monomial symmetric function with exponents given by the parts of $\lambda$, in indeterminates $x_{1}, x_{2}, \ldots$, by $m_{\lambda}$. In this paper, we consider the particular evaluation of the monomial symmetric function at $x_{i}=i$, for $i=1, \ldots, k-1$, and $x_{i}=0$, for $i \geq k$, and write this as $\hat{m}_{\lambda}$. Now let $C(t)=\sum_{m \geq 0} C_{m} t^{m}$, so from (1.5) we obtain

$$
C(t)=\frac{1}{1-\sum_{i \geq 2}(i-1) R_{i} t^{i}} .
$$

Let $D=t \frac{d}{d t}, I$ be the identity operator, and define

$$
P_{m}(t)=-\frac{1}{m !} C(t)(D+(m-2) I) C(t) \cdots(D+I) C(t) D C(t), \quad m \geq 1 .
$$

For example, we have

$$
P_{1}(t)=-C(t), \quad P_{2}(t)=-\frac{1}{2} C(t) D C(t),
$$

and

$$
\begin{aligned}
P_{3}(t) & =-\frac{1}{6} C(t)(D+I) C(t) D C(t) \\
& =\frac{1}{6}\left(C(t)^{2} D C(t)+C(t)(D C(t))^{2}+C(t)^{2} D^{2} C(t)\right) .
\end{aligned}
$$


We remind the reader that the differential operator is not commutative; for example, $(D C(t))^{2} \neq D C(t) D C(t)$. The latter is properly interpreted as $D(C(t) D C(t))$. Finally, for a partition $\lambda$, we write $P_{\lambda}(t)=\prod_{j=1}^{l(\lambda)} P_{\lambda_{j}}(t)$. We now state our main result.

Theorem 2.1. For $n \geq 1, k \geq 2 n-1$,

$$
\Sigma_{k, 2 n}=-\frac{1}{k}\left[t^{k+1-2 n}\right] \sum_{\lambda \vdash 2 n} \hat{m}_{\lambda} \frac{P_{\lambda}(t)}{C(t)} .
$$

There is a slight modification of this result, given below, in which the term corresponding to the partition with one part is given a simpler (but equivalent) evaluation. This is used mainly for computational purposes, as it is easier to compute $P_{i}$ for smaller $i$.

Theorem 2.2. For $n \geq 1, k \geq 2 n-1$,

$$
\Sigma_{k, 2 n}=-\frac{1}{k}\left[t^{k+1-2 n}\right]\left(\frac{k-1}{2 n} \hat{m}_{2 n} P_{2 n-1}(t)+\sum_{\substack{\lambda \vdash 2 n \\ l(\lambda) \geq 2}} \hat{m}_{\lambda} \frac{P_{\lambda}(t)}{C(t)}\right) .
$$

The following result gives a generating function form of the main result.

Theorem 2.3. For $n \geq 1, k \geq 2 n-1$,

$$
\begin{gathered}
\Sigma_{k, 2 n}=-\frac{1}{k}\left[u^{2 n} t^{k+1}\right] \frac{1}{C(t)} \prod_{j=1}^{k-1}\left(1+\sum_{i \geq 1} j^{i} P_{i}(t) u^{i} t^{i}\right) \\
\Sigma_{k}=-\frac{1}{k}\left[t^{k+1}\right] \frac{1}{C(t)} \prod_{j=1}^{k-1}\left(1+\sum_{i \geq 1} j^{i} P_{i}(t) t^{i}\right) .
\end{gathered}
$$

Note that, for each $n \geq 1$, these results give $\Sigma_{k, 2 n}$ as the coefficient of $t^{k+1-2 n}$ in a polynomial in $C(t)$ and

$$
D^{i} C(t)=\sum_{m \geq 2} m^{i} C_{m} t^{m}, \quad i \geq 1
$$

Thus $\Sigma_{k, 2 n}$ is written as a polynomial in the $C_{m}$ 's, with coefficients that are polynomial in $k$ with rational coefficients, so our results give C-expansions for $\Sigma_{k, 2 n}$, for $n \geq 1$ (the $\hat{m}_{\lambda}$ are divisible by $k$ for each $\lambda$ and fixed $n$, as follows immediately from Propositions 3.1 and 3.2. .

Using the above results, with the help of Maple, we have determined the Cexpansions and the R-expansions of $\Sigma_{k, 2 n}$ for all $k \leq 25$ and $n \geq 1$. The Rexpansions are in complete agreement with those reported in Biane [3] for $k \leq 11$. 
The C-expansions are given below for $k \leq 10$ :

$$
\begin{aligned}
\Sigma_{1}-R_{2} & =0 \\
\Sigma_{2}-R_{3} & =0 \\
\Sigma_{3}-R_{4} & =C_{2}, \\
\Sigma_{4}-R_{5} & =\frac{5}{2} C_{3}, \\
\Sigma_{5}-R_{6} & =5 C_{4}+8 C_{2}, \\
\Sigma_{6}-R_{7} & =\frac{35}{4} C_{5}+42 C_{3}, \\
\Sigma_{7}-R_{8} & =14 C_{6}+\frac{469}{3} C_{4}+\frac{203}{3} C_{2}^{2}+180 C_{2}, \\
\Sigma_{8}-R_{9} & =21 C_{7}+\frac{1869}{4} C_{5}+\frac{819}{2} C_{3} C_{2}+1522 C_{3}, \\
\Sigma_{9}-R_{10} & =30 C_{8}+1197 C_{6}+\frac{963}{2} C_{3}{ }^{2}+1122 C_{4} C_{2}+81 C_{2}^{3}+\frac{26060}{3} C_{4} \\
& +\frac{17680}{3} C_{2}^{2}+8064 C_{2}, \\
\Sigma_{10}-R_{11} & =\frac{165}{4} C_{9}+\frac{5467}{2} C_{7}+\frac{4433}{2} C_{4} C_{3}+\frac{1133}{2} C_{3} C_{2}{ }^{2}+\frac{11033}{4} C_{5} C_{2} \\
& +38225 C_{5}+52580 C_{3} C_{2}+96624 C_{3} .
\end{aligned}
$$

Note the form of the data presented above. We have

$$
\Sigma_{k}-\Sigma_{k, 0}=\sum_{k \geq 1} \Sigma_{k, 2 n}
$$

where $\Sigma_{k, 0}=R_{k+1}$ remains on the left hand side, and we can recover the individual $\Sigma_{k, 2 n}$ on the right hand side: if the weight of the monomial $C_{m_{1}} \ldots C_{m_{i}}$ is $m_{1}+$ $\cdots+m_{i}$, then, from (1.5) and (1.4), $\Sigma_{k, 2 n}$ is the sum of all terms of weight $k+1-2 n$.

In the above C-expansions for $k \leq 10$, all nonzero coefficients are positive rationals, with apparently small denominators. In fact, this is true for all the data we have computed, up to $k=25$. We do not have a precise conjecture about the denominators, but conjecture that the positivity holds for all $k$.

Conjecture 2.4. For $n \geq 1, k \geq 2 n-1, \Sigma_{k, 2 n}$ is $C$-positive.

This C-positivity conjecture implies the R-positivity conjecture, from (1.5) (so, our data also check the R-positivity conjecture for $k \leq 25$ ). Theorem 1.3 gives an immediate proof that Conjecture 2.4 holds for $n=1$ and all $k$. In Corollary 3.5. we are able to prove that Conjecture 2.4 holds for $n=2$ and all $k$. We are not able to prove the conjecture for any larger value of $n$, though of course Theorem 1.4. together with (2.1), proves that the linear terms are C-positive for all $n$.

The conjecture does not hold for $n=0$, as described below. We have $\Sigma_{k, 0}=$ $R_{k+1}$, and it is straightforward to determine the C-expansion for the $R_{i}$ 's: from (2.1) we obtain

$$
\begin{aligned}
1-\sum_{i \geq 2}(i-1) R_{i} t^{i} & =\frac{1}{C(t)} \\
& =\sum_{j_{2}, j_{3}, \ldots \geq 0}\left(j_{2}+j_{3}+\cdots\right) ! \prod_{m \geq 2} \frac{\left(-C_{m} t^{m}\right)^{j_{m}}}{j_{m} !},
\end{aligned}
$$

so we conclude that

$$
R_{i}=\frac{1}{i-1} \sum_{\substack{j_{2}, j_{3}, \ldots \geq 0 \\ 2 j_{2}+3 j_{3}+\cdots=i}}(-1)^{1+j_{2}+j_{3}+\cdots}\left(j_{2}+j_{3}+\cdots\right) ! \prod_{m \geq 2} \frac{C_{m}^{j_{m}}}{j_{m} !}, \quad i \geq 2 .
$$


Thus, terms of negative sign appear in the C-expansion of $R_{i}$, for $i \geq 4$. This is the reason that we have presented the data for $k$ up to 10 with $R_{k+1}$ subtracted on the left hand side. This is also the reason that the $\mathrm{R}$-positivity conjecture does not imply the C-positivity conjecture, so R-positivity and C-positivity are not equivalent.

\section{Special CASES of the main Result}

3.1. Monomial symmetric functions. To make the expression for $\Sigma_{k, 2 n}$ that arises from Theorem 2.1 (or Theorem 2.2) explicit, we need to evaluate the $\hat{m}_{\lambda}$, which are monomial symmetric functions in $1,2, \ldots, k-1$. For general results about symmetric functions, see Macdonald [10].

Proposition 3.1. For indeterminates $a_{i}, i \geq 1$, let $A(x)=1+\sum_{i \geq 1} a_{i} x^{i}$, and $a_{\lambda}=\prod_{j=1}^{l(\lambda)} a_{\lambda_{j}}$, where $\lambda=\lambda_{1} \ldots \lambda_{l(\lambda)}$ is a partition. Then

$$
\sum_{\lambda \in \mathcal{P}} \hat{m}_{\lambda} a_{\lambda}=\exp \sum_{j \geq 1} \hat{m}_{j} \sum_{i \geq 1} \frac{(-1)^{i-1}}{i}\left[x^{j}\right](A(x)-1)^{i} .
$$

Proof. We have

$$
\begin{aligned}
\sum_{\lambda \in \mathcal{P}} m_{\lambda} a_{\lambda} & =\prod_{n \geq 1} A\left(x_{n}\right) \\
& =\exp \sum_{n \geq 1} \log \left(A\left(x_{n}\right)\right) \\
& =\exp \sum_{n \geq 1} \sum_{i \geq 1} \frac{(-1)^{i-1}}{i}\left(A\left(x_{n}\right)-1\right)^{i},
\end{aligned}
$$

and the result follows.

Proposition 3.1 gives an expression for $\hat{m}_{\lambda}$ as a polynomial in $\hat{m}_{i}, i \geq 1$, by equating coefficients of $a_{\lambda}$. To evaluate the $\hat{m}_{i}, i \geq 1$, we apply the following result (see, e.g., [10, I 2, Exercise 11] for a proof).

Proposition 3.2. For $j \geq 1$,

$$
\hat{m}_{j}=\sum_{i=1}^{j} S(j, i) i !\left(\begin{array}{c}
k \\
i+1
\end{array}\right)
$$

where $S(j, i)$, the Stirling numbers of the second kind, are given by

$$
\sum_{i \geq 0} \sum_{j=0}^{i} S(j, i) u^{i} \frac{x^{j}}{j !}=\exp u\left(e^{x}-1\right)
$$

As special cases of this result, we have the following, well-known sums of integer powers:

$$
\begin{gathered}
\hat{m}_{1}=\frac{1}{2}(k-1) k, \quad \hat{m}_{2}=\frac{1}{6}(k-1) k(2 k-1), \quad \hat{m}_{3}=\frac{1}{4}(k-1)^{2} k^{2}, \\
\hat{m}_{4}=\frac{1}{30}(k-1) k(2 k-1)\left(3 k^{2}-3 k-1\right) .
\end{gathered}
$$


3.2. The cases $n=1,2$. We first consider the case $n=1$ of Theorem 2.2. This immediately gives Biane and Śniady's C-expansion for $\Sigma_{k, 2}$, and hence another proof of Theorem 1.3, as shown below.

Proof of Theorem 1.3. From Theorem 2.2, with $n=1$, we obtain

$$
\begin{aligned}
\Sigma_{k, 2} & =-\frac{1}{k}\left[t^{k-1}\right]\left(-\frac{1}{2}(k-1) \hat{m}_{2} C(t)+\hat{m}_{11} C(t)\right) \\
& =\frac{1}{k}\left(\frac{1}{2}(k-1) \hat{m}_{2}-\hat{m}_{11}\right)\left[t^{k-1}\right] C(t) .
\end{aligned}
$$

But from Proposition 3.1, we obtain

$$
\hat{m}_{11}=\frac{1}{2}\left(\hat{m}_{1}^{2}-\hat{m}_{2}\right),
$$

and the result follows from (3.1), by routine manipulation.

Next we consider the case $n=2$ of Theorem 2.2, to obtain an explicit Cexpansion for $\Sigma_{k, 4}$.

Theorem 3.3. For $k \geq 3$,

$$
\Sigma_{k, 4}=\alpha(k) \sum_{\substack{i, j, m \geq 0 \\ i+j+m=k-3}} C_{i} C_{j} C_{m}+\beta(k) \sum_{\substack{i, j, m \geq 0 \\ i+j+m=k-3}} i^{2} C_{i} C_{j} C_{m},
$$

where

$$
\begin{aligned}
& \alpha(k)=-\frac{1}{17280}(k-3)(k-1)^{2} k(k+1)\left(k^{2}-4 k-6\right), \\
& \beta(k)=\frac{1}{2880}(k-1) k(k+1)\left(2 k^{2}-3\right) .
\end{aligned}
$$

Proof. From Theorem 2.2, with $n=2$, letting $b=\frac{1}{6}\left(\hat{m}_{31}-\frac{1}{4}(k-1) \hat{m}_{4}\right)$, we obtain

$$
\begin{aligned}
\Sigma_{k, 4} & =-\frac{1}{k}\left[t^{k-3}\right]\left(b\left(C(t)^{2} D C(t)+C(t)(D C(t))^{2}+C(t)^{2} D^{2} C(t)\right)\right. \\
& \left.+\frac{1}{4} \hat{m}_{22} C(t)(D C(t))^{2}-\frac{1}{2} \hat{m}_{211} C(t)^{2} D C(t)+\hat{m}_{1111} C(t)^{3}\right) \\
& =-\frac{1}{k}\left[t^{k-3}\right]\left(\hat{m}_{1111} C(t)^{3}+\left(b-\frac{1}{2} \hat{m}_{211}\right) C(t)^{2} D C(t)\right. \\
& \left.+b C(t)^{2} D^{2} C(t)+\left(b+\frac{1}{4} \hat{m}_{22}\right) C(t)(D C(t))^{2}\right) \\
& =-\frac{1}{k}\left[t^{k-3}\right]\left(\hat{m}_{1111} C(t)^{3}+\left(b-\frac{1}{2} \hat{m}_{211}\right) \frac{1}{3} D C(t)^{3}\right. \\
& \left.+b C(t)^{2} D^{2} C(t)+\left(b+\frac{1}{4} \hat{m}_{22}\right)\left(\frac{1}{6} D^{2} C(t)^{3}-\frac{1}{2} C(t)^{2} D^{2} C(t)\right)\right) \\
& =-\frac{1}{k}\left(\hat{m}_{1111}+\frac{1}{3}(k-3)\left(b-\frac{1}{2} \hat{m}_{211}\right)+\frac{1}{6}(k-3)^{2}\left(b+\frac{1}{4} \hat{m}_{22}\right)\right)\left[t^{k-3}\right] C(t)^{3} \\
& -\frac{1}{k}\left(\frac{1}{2} b-\frac{1}{8} \hat{m}_{22}\right)\left[t^{k-3}\right] C(t)^{2} D^{2} C(t) .
\end{aligned}
$$

But from Proposition 3.1, we obtain

$$
\begin{aligned}
\hat{m}_{31} & =\hat{m}_{3} \hat{m}_{1}-\hat{m}_{4}, \\
\hat{m}_{22} & =\frac{1}{2}\left(\hat{m}_{2}^{2}-\hat{m}_{4}\right), \\
\hat{m}_{211} & =\frac{1}{2}\left(\hat{m}_{2} \hat{m}_{1}^{2}-2 \hat{m}_{3} \hat{m}_{1}-\hat{m}_{2}^{2}+2 \hat{m}_{4}\right), \\
\hat{m}_{1111} & =\frac{1}{24}\left(\hat{m}_{1}^{4}-6 \hat{m}_{2} \hat{m}_{1}^{2}+8 \hat{m}_{3} \hat{m}_{1}+3 \hat{m}_{2}^{2}-6 \hat{m}_{4}\right),
\end{aligned}
$$


so from (3.1), by routine manipulation, we obtain

$$
\Sigma_{k, 4}=\alpha(k)\left[t^{k-3}\right] C(t)^{3}+\beta(k)\left[t^{k-3}\right] C(t)^{2} D^{2} C(t),
$$

where $\alpha(k)$ and $\beta(k)$ are given above. The result follows.

For monomials in $R_{2}, R_{3}, \ldots$ that are pure powers of a single $R_{m}$, we have the following form of the above result.

Corollary 3.4. For $m \geq 2, i \geq 1$,

$$
\begin{aligned}
{\left[R_{m}^{i}\right] \Sigma_{m i+3,4} } & =\frac{1}{34560}(m-1)^{i} m i(i+1)(i+2)(m i+2)(m i+3)(m i+4) \\
& \times\left(m^{3} i^{3}+2 m^{2}(m+4) i^{2}+4 m(3 m+5) i+15 m+18\right) .
\end{aligned}
$$

Proof. From Theorem 3.3, we obtain

$$
\left[R_{m}^{i}\right] \Sigma_{m i+3,4}=\alpha(m i+3)\left[R_{m}^{i} t^{m i}\right] C(t)^{3}+\beta(m i+3)\left[R_{m}^{i} t^{m i}\right] C(t)^{2} D^{2} C(t) .
$$

Now, setting $R_{j}=0$ for $j \neq m$, we obtain $C(t)=\left(1-(m-1) R_{m} t^{m}\right)^{-1}$, so

$$
\left[R_{m}^{i} t^{m i}\right] C(t)^{3}=(m-1)^{i}\left(\begin{array}{c}
i+2 \\
2
\end{array}\right) .
$$

Also, we have

$$
\begin{aligned}
D^{2} C(t)= & D m(m-1) R_{m} t^{m}\left(1-(m-1) R_{m} t^{m}\right)^{-2} \\
= & D m\left(\left(1-(m-1) R_{m} t^{m}\right)^{-2}-\left(1-(m-1) R_{m} t^{m}\right)^{-1}\right) \\
= & m^{2}(m-1)\left(2 R_{m} t^{m}\left(1-(m-1) R_{m} t^{m}\right)^{-3}\right. \\
& \left.\quad-R_{m} t^{m}\left(1-(m-1) R_{m} t^{m}\right)^{-2}\right),
\end{aligned}
$$

so

$$
\left[R_{m}^{i} t^{m i}\right] C(t)^{2} D^{2} C(t)=(m-1)^{i} m^{2}\left(2\left(\begin{array}{c}
i+3 \\
4
\end{array}\right)-\left(\begin{array}{c}
i+2 \\
3
\end{array}\right)\right) .
$$

The result follows by routine manipulation.

We now consider the case $m=2$ of Corollary 3.4, to obtain an immediate proof of Stanley's Conjecture 1.5 .

Proof of Conjecture 1.5. We set $m=2$ in Corollary 3.4. Then the factor that is cubic in $i$ becomes

$$
8 i^{3}+48 i^{2}+88 i+48=8(i+1)(i+2)(i+3),
$$

and the result follows.

As the final result of this section, we are able to use the explicit C-expansion given in Theorem 3.3, to prove the C-positivity of $\Sigma_{k, 4}$.

Corollary 3.5. $\Sigma_{k, 4}$ is $C$-positive for all $k \geq 3$. 
Proof. Consider $0 \leq i \leq j \leq m$, with $i+j+m=k-3$, and let $\gamma=|\operatorname{Aut}(i, j, m)|$. Thus when $k=12$, for example, $\gamma=1$ for $(i, j, m)=(2,3,4)$ or $(0,2,7), \gamma=2$ for $(i, j, m)=(2,2,5)$ or $(1,4,4)$, and $\gamma=6$ for $(i, j, m)=(3,3,3)$. Then, from Theorem 3.3, we obtain

$$
\left[C_{i} C_{j} C_{m}\right] \Sigma_{k, 4}=\frac{6}{\gamma} \alpha(k)+\frac{2}{\gamma}\left(i^{2}+j^{2}+m^{2}\right) \beta(k) .
$$

Now, the minimum value of $x^{2}+y^{2}+z^{2}$ over the reals, subject to $x+y+z=c$, for any fixed real $c$, is achieved at $x=y=z=c / 3$, so in the above expression we have $i^{2}+j^{2}+m^{2} \geq \frac{1}{3}(k-3)^{2}$. But $\beta(k)>0$ for $k \geq 3$, so we obtain

$$
\begin{aligned}
& {\left[C_{i} C_{j} C_{m}\right] \Sigma_{k, 4} \geq \frac{2}{\gamma}\left(3 \alpha(k)+\frac{1}{3}(k-3)^{2} \beta(k)\right) } \\
= & \frac{1}{8640 \gamma}(k-3)(k-1) k(k+1)\left(-3(k-1)\left(k^{2}-4 k-6\right)+2(k-3)\left(2 k^{2}-3\right)\right) \\
= & \frac{1}{8640 \gamma}(k-3)(k-1) k^{3}(k+1)(k+3) \geq 0,
\end{aligned}
$$

for $k \geq 3$, giving the result.

3.3. The linear terms. We now apply Theorem 2.3 to evaluate the linear terms in $\Sigma_{k}$, and thus obtain another proof of Theorem 1.4,

Proof of Theorem 1.4. For $i \geq 1$, let $A^{(i)}(t)$ consist of the terms in $P_{i}(t)$ that are linear in the $C_{m}$ 's. Also, let $L_{n, k}=\left[R_{k+1-2 n}\right] \Sigma_{k, 2 n}$. We apply Theorem 2.3 to determine $L_{n, k}$. From (2.1), we have

$$
\begin{aligned}
L_{n, k} & =\left[\frac{C_{k+1-2 n}}{k-2 n}\right] \Sigma_{k, 2 n}=\left[\frac{C_{k+1-2 n}}{k-2 n}\right] \Sigma_{k} \\
& =-\frac{1}{k}\left[\frac{C_{k+1-2 n}}{k-2 n} t^{k+1}\right] \frac{1}{C(t)} \prod_{j=1}^{k-1}\left(1-j t+\sum_{i \geq 1} j^{i} A^{(i)}(t) t^{i}\right) \\
& =-\frac{1}{k}\left[\frac{C_{k+1-2 n}}{k-2 n} t^{k+1}\right] \frac{1}{C(t)}\left(\prod_{j=1}^{k-1}\left(1+\sum_{i \geq 1} \frac{j^{i} A^{(i)}(t) t^{i}}{1-j t}\right)\right) \prod_{a=1}^{k-1}(1-a t) \\
& =-\frac{1}{k}\left[\frac{C_{k+1-2 n}}{k-2 n} t^{k+1}\right]\left(1-C(t)+\sum_{j=1}^{k-1} \sum_{i \geq 1} \frac{j^{i} A^{(i)}(t) t^{i}}{1-j t}\right) \prod_{a=1}^{k-1}(1-a t) .
\end{aligned}
$$

But

$A^{(i)}(t)=-\frac{1}{i !}(D+(i-2) I) \cdots(D+I) D C(t)=-\sum_{m \geq 2}\left(\begin{array}{c}-(m-1) \\ i\end{array}\right)(-1)^{i} \frac{C_{m}}{m-1} t^{m}$,

for $i \geq 1$. Now let $\frac{C_{m}}{m-1}=x^{m-1}, m \geq 2$, which gives

$$
\begin{aligned}
\sum_{i \geq 1} j^{i} A^{(i)}(t) t^{i} & =-\sum_{m \geq 2}\left((1-j t)^{-(m-1)}-1\right) x^{m-1} t^{m} \\
& =-\frac{t}{1-\frac{x t}{1-j t}}+\frac{t}{1-x t}
\end{aligned}
$$

and

$$
1-C(t)=-\sum_{m \geq 2}(m-1) x^{m-1} t^{m}=-\frac{t}{(1-x t)^{2}}+\frac{t}{1-x t} .
$$


Thus we obtain

$$
\begin{aligned}
L_{n, k}=\frac{1}{k}\left[x^{k-2 n} t^{k+1}\right] & \left(\frac{t}{(1-x t)^{2}}-\frac{t}{1-x t}\right. \\
+ & \left.\sum_{j=1}^{k-1}\left(\frac{t}{1-(j+x) t}-\frac{t}{(1-j t)(1-x t)}\right)\right) \prod_{a=1}^{k-1}(1-a t) .
\end{aligned}
$$

We now finish the proof using the method of Biane [3, Theorem 6.1]: Replace $t$ by $t^{-1}$, and multiply by $t^{k}$, to obtain

$$
\begin{aligned}
L_{n, k}=\frac{1}{k}\left[x^{k-2 n}\right]\left[t^{-1}\right]_{\infty}(t)_{k} & \frac{t}{(t-x)^{2}}-\frac{1}{t-x} \\
& \left.+\sum_{j=1}^{k-1}\left(\frac{1}{t-j-x}-\frac{t}{(t-j)(t-x)}\right)\right)
\end{aligned}
$$

where $(t)_{k}=t(t-1) \cdots(t-k+1)$ is the falling factorial. Now use the fact that the residue is unchanged if we substitute $t+c$ for $t$, where $c$ is independent of $t$. Thus, substituting $t+j+x$ for $t$ in the first term of the summation over $j$, and substituting $t+x$ for $t$ in all other terms, we obtain

$$
\begin{aligned}
L_{n, k} & =\frac{1}{k}\left[x^{k-2 n}\right]\left([t](t+x)(t+x)_{k}-(x)_{k}+\sum_{j=1}^{k-1}\left((x+j)_{k}-\frac{x(x)_{k}}{x-j}\right)\right) \\
& =\frac{1}{k}\left[x^{k-2 n}\right] \sum_{j=0}^{k-1}(x+j)_{k}=\frac{1}{k}\left[x^{k-2 n}\right] \sum_{j=0}^{k-1}(x-j)_{k},
\end{aligned}
$$

where, for the last equality, we have replaced $x$ by $-x$, and multiplied by $(-1)^{k}$. The result now follows, as shown in Biane [3].

\section{LAGRANGE INVERSION AND THE PROOF OF THE MAIN RESUlT}

As a first step, we translate Theorem 1.2 into the language of formal power series, using the notation

$$
\phi(x)=x H\left(x^{-1}\right), \quad \Phi(x, u)=\sum_{i \geq 0} \Phi_{i}(x) u^{i}=(1-u x) \phi\left(x(1-u x)^{-1}\right),
$$

where $H(x)$ is defined in (1.3).

Proposition 4.1. The following two equations hold:

1) For $k \geq 1$

$$
\Sigma_{k}=-\frac{1}{k}\left[x^{k+1}\right] \prod_{j=0}^{k-1} \Phi(x, j) .
$$

2) For $k, n \geq 1$,

$$
\Sigma_{k, 2 n}=-\frac{1}{k}\left[u^{2 n} x^{k+1}\right] \prod_{j=0}^{k-1} \Phi(x, j u) .
$$


Proof. For (4.2), we first replace $x$ by $x^{-1}$ in Theorem 1.2, to obtain

$$
\Sigma_{k}=-\frac{1}{k}\left[x^{k+1}\right] \prod_{j=0}^{k-1} x H\left(x^{-1}(1-j x)\right)
$$

and the result follows immediately.

For (4.3), we let $\vartheta$ be the substitution operator $R_{i} \mapsto u^{i} R_{i}, i \geq 2$. Then, from (1.4), we have

$$
\Sigma_{k, 2 n}=\left[u^{k+1-2 n}\right] \vartheta \Sigma_{k} .
$$

Now, from (1.3), we have

$$
\vartheta F(x)=\frac{x}{\vartheta R(x)}=\frac{x}{R(u x)}=\frac{1}{u} F(u x) .
$$

Applying $\vartheta$ to both sides of the equation $x=F\left(F^{\langle-1\rangle}(x)\right)$ we obtain

$$
\begin{aligned}
x & =\vartheta F\left(\vartheta F^{\langle-1\rangle}(x)\right) \\
& =\frac{1}{u} F\left(u \vartheta F^{\langle-1\rangle}(x)\right),
\end{aligned}
$$

implying

$$
\vartheta F^{\langle-1\rangle}(x)=\frac{1}{u} F^{\langle-1\rangle}(u x) .
$$

Thus, combining this with (1.3) and (4.1), we obtain

$$
\vartheta \phi(x)=x \vartheta H\left(x^{-1}\right)=\frac{x}{\vartheta F^{\langle-1\rangle}(x)}=\frac{u x}{F^{\langle-1\rangle}(u x)}=\phi(u x),
$$

and then

$$
\vartheta \Phi(x, j)=(1-j x) \phi\left(u x(1-j x)^{-1}\right)=\Phi\left(u x, j u^{-1}\right) .
$$

Combining this with (4.4) and (4.2) gives

$$
\Sigma_{k, 2 n}=-\frac{1}{k}\left[u^{k+1-2 n} x^{k+1}\right] \prod_{j=0}^{k-1} \Phi\left(u x, j u^{-1}\right),
$$

and (4.3) now follows, by substituting first $x=x u^{-1}$, and then $u=u^{-1}$.

Next, we give an expression for the coefficients $\Phi_{i}, i \geq 0$, defined in (4.1).

Proposition 4.2. For $i \geq 0$,

$$
\Phi_{i}(x)=\frac{x}{i !}\left(x^{2} \frac{d}{d x}\right)^{i} \frac{\phi(x)}{x} .
$$

Note that for $i=0$, this specializes to $\Phi_{0}(x)=\phi(x)$.

Proof. From (1.3) and (4.1), we have

$$
\phi(x)=1+\sum_{j \geq 2} \phi_{j} x^{j},
$$


where $\phi_{j}, j \geq 2$, are polynomials in the $R_{i}$ 's. For $i=0$, we have $\Phi_{0}(x)=\Phi(x, 0)=$ $\phi(x)$. For $i \geq 1$, we have

$$
\begin{aligned}
\Phi_{i}(x) & =\left[u^{i}\right] \Phi(x, u)=\left[u^{i}\right]\left(1-u x+\sum_{j \geq 2} \phi_{j} x^{j}(1-u x)^{1-j}\right) \\
& =-\left(\begin{array}{c}
1 \\
i
\end{array}\right) x+\sum_{j \geq 2} \phi_{j}\left(\begin{array}{c}
j+i-2 \\
i
\end{array}\right) x^{j+i} \\
& =\frac{x}{i !}\left(x^{2} \frac{d}{d x}\right)^{i}\left(\frac{1}{x}+\sum_{j \geq 2} \phi_{j} x^{j-1}\right),
\end{aligned}
$$

and the result follows.

We make use of the following two, closely related, versions of Lagrange's Theorem (see, e.g., [6, Section 1.2], for a proof).

Theorem 4.3. Suppose $\phi$ is a formal power series with invertible constant term. Then the functional equation $w=t \phi(w)$ has a unique formal power series solution $w=w(t)$. Moreover,

1) For a formal Laurent series $f$ and $n \neq 0$, we have

$$
\frac{1}{n}\left[x^{n-1}\right]\left(\frac{d}{d x} f(x)\right) \phi(x)^{n}=\left[t^{n}\right] f(w),
$$

2) For a formal power series $f$, and $n \geq 0$, we have

$$
\left[x^{n}\right] f(x) \phi(x)^{n}=\left[t^{n}\right] f(w) \frac{t}{w} \frac{d w}{d t} .
$$

Here, we shall consider the functional equation

$$
w=t \phi(w),
$$

where $\phi$ is the particular series given by (4.1). Then from (1.3) and (4.1), we have

$$
w=t w H\left(w^{-1}\right)=\frac{t w}{F^{\langle-1\rangle}(w)},
$$

so $F^{\langle-1\rangle}(w)=t$, and from (1.3) we deduce that

$$
t=w R(t) .
$$

We now relate the series $C(t)$ and differential operator $D$ of Section 2 to the variable $w$.

\section{Proposition 4.4.}

$$
\begin{aligned}
& \frac{D w}{w}=\frac{1}{R(t) C(t)}, \\
& w^{2} \frac{d}{d w}=t C(t) D .
\end{aligned}
$$


Proof. From (2.1) and (1.3), we obtain

$$
C(t)=\frac{1}{-t D \frac{R(t)}{t}}
$$

But

$$
\frac{D w}{w}=-w D \frac{1}{w}=-\frac{t}{R(t)} D \frac{R(t)}{t},
$$

from (4.7), and result (4.8) follows.

Now, (4.8) gives the operator identity

$$
w \frac{d}{d w}=R(t) C(t) D
$$

and multiplying by $w$ and using (4.7), we obtain result (4.9).

Proof of Theorem 2.1. For a partition $\lambda$, let $\Phi_{\lambda}(x)=\prod_{j=1}^{l(\lambda)} \Phi_{\lambda_{j}}(x)$. Then from (4.3) and (4.5), we have

$$
\begin{aligned}
\Sigma_{k, 2 n} & =-\frac{1}{k}\left[x^{k+1}\right] \sum_{\lambda \vdash 2 n} \hat{m}_{\lambda} \Phi_{\lambda}(x) \phi(x)^{k-l(\lambda)} \\
& =-\frac{1}{k}\left[x^{k+1}\right] \sum_{\lambda \vdash 2 n} \hat{m}_{\lambda} \frac{\Phi_{\lambda}(x)}{\phi(x)^{l(\lambda)+1}} \phi(x)^{k+1} \\
& =-\frac{1}{k}\left[t^{k+1}\right] \sum_{\lambda \vdash 2 n} \hat{m}_{\lambda} \frac{1}{R(t) C(t)} \frac{\Phi_{\lambda}(w)}{\phi(w)^{l(\lambda)+1}},
\end{aligned}
$$

where the last equality follows from 2) of Theorem 4.3 and (4.8). But, from (4.5), (4.6) and (4.9), for $i \geq 1$ we have

$$
\begin{aligned}
\frac{\Phi_{i}(w)}{\phi(w)} & =\frac{1}{i !} \frac{w}{\phi(w)}\left(w^{2} \frac{d}{d w}\right)^{i} \frac{\phi(w)}{w} \\
& =\frac{t}{i !}(t C(t) D)^{i-1} t C(t) D \frac{1}{t} \\
& =-\frac{t}{i !}(t C(t) D)^{i-1} C(t) .
\end{aligned}
$$

Finally, we prove by induction on $i \geq 1$ that

$$
-\frac{1}{i !}(t C(t) D)^{i-1} C(t)=t^{i-1} P_{i}(t)
$$

where $P_{i}(t)$ is defined in Section 2. The result is clearly true for $i=1$. For the induction step, we have

$$
\begin{aligned}
-\frac{1}{(i+1) !}(t C(t) D)^{i} C(t) & =\frac{1}{i+1} t C(t) D t^{i-1} P_{i}(t) \\
& =\frac{1}{i+1}\left(t^{i} C(t) D+(i-1) t^{i} C(t) I\right) P_{i}(t) \\
& =t^{i} P_{i+1}(t),
\end{aligned}
$$

as required. Together, these results give

$$
\frac{\Phi_{i}(w)}{\phi(w)}=t^{i} P_{i}(t),
$$


SO

$$
\frac{\Phi_{\lambda}(w)}{\phi(w)^{l(\lambda)+1}}=t^{2 n} \frac{P_{\lambda}(t)}{\phi(w)},
$$

since $\lambda \vdash 2 n$, and the result follows from (4.6) and (4.7).

Proof of Theorem 2.2. In the proof of Theorem 2.1, the term in $\Sigma_{k, 2 n}$ corresponding to the partition with the single part $2 n$ can be treated in the following modified way. We obtain

$$
\begin{gathered}
-\frac{1}{k}\left[x^{k+1}\right] \hat{m}_{2 n} \Phi_{2 n}(x) \phi(x)^{k-1}=-\frac{1}{k}\left[x^{k-2}\right] \hat{m}_{2 n} x^{-3} \Phi_{2 n}(x) \phi(x)^{k-1} \\
=-\frac{1}{k}\left[x^{k-2}\right] \hat{m}_{2 n} x^{-3} \phi(x)^{k-1} \frac{x}{(2 n) !} x^{2} \frac{d}{d x}\left(x^{2} \frac{d}{d x}\right)^{2 n-1} \frac{\phi(x)}{x} \\
=-\frac{k-1}{k}\left[t^{k-1}\right] \hat{m}_{2 n} \frac{1}{(2 n) !}\left(w^{2} \frac{d}{d w}\right)^{2 n-1} \frac{\phi(w)}{w}
\end{gathered}
$$

from 1) of Theorem 4.3, and the result follows as in the above proof of Theorem 2.1

\section{ACKNOWLEDGEMENTS}

This work was supported by a Discovery Grant from NSERC (IG), a Postgraduate Scholarship from NSERC (AR), and an Ontario Graduate Scholarship in Science and Technology (AR). We would like to thank P. Biane, A. Okounkov, P. Śniady and R. Stanley for helpful comments on an earlier draft.

\section{REFERENCES}

1. P. Biane, Representations of symmetric groups and free probability, Advances in Mathematics 138 (1998), 126-181. MR.1644993 (2001b:05225)

2. _ Free cumulants and representations of large symmetric groups, Proceedings of the XIIIth International Congress of Mathematical Physics, London, Int. Press (2000), 321-326. MR:1883322 (2002k:20020)

3. Characters of symmetric groups and free cumulants, Asymptotic Combinatorics with Applications to Mathematical Physics, A. Vershik (Ed.), Springer Lecture Notes in Mathematics 1815 (2003), 185-200. MR2009840 (2004j:20016)

4. S. Corteel, A. Goupil, and G. Schaeffer, Content evaluation and class symmetric functions, Adv. in Maths (2004), no. 188, 315-336. MR2087230 (2005e:05150)

5. A. Frumkin, G. James, and Y. Roichman, On trees and characters, J. Algebraic Combinatorics 17 (2003), 323-334. MR2001674 (2005i:05196)

6. I.P. Goulden and D.M. Jackson, Combinatorial enumeration, Wiley-Interscience, New York, 1983 (Dover Reprint, 2004). MR2079788(2005b:05001)

7. V. Ivanov and G. Olshanski, Kerov's central limit theorem for the Plancherel measure on Young diagrams, Symmetric functions 2001: Surveys of developments and perspectives, S. Fomin (Ed.), NATO Science series II. Mathematics, Physics and Chemistry 74 (2002), 93-151.

8. J. Katriel, Explicit expressions for the central characters of the symmetric group, Discrete Applied Math. 67 (1996), 149-156. MR1393301 (97e:20013)

9. S. Kerov, Gaussian limit for the Plancherel measure of the symmetric group, Comptes Rendus Acad. Sci. Paris, Série I 316 (1993), 303-308. MR1204294 (93k:20106)

10. I.G. Macdonald, Symmetric functions and Hall polynomials, second ed., Oxford University Press, Oxford, 1995. MR,1354144 (96h:05207) 
11. P. Śniady, Asymptotics of characters of symmetric groups and free probability, Discrete Math 306 (2006), 624-665. MR2215589

12. R.P. Stanley, Kerov's character polynomial and irreducible symmetric group characters of rectangular shape, Transparencies from a talk at CMS meeting, Quebec City, 2002.

Department of Combinatorics and Optimization, University of Waterloo, Waterloo, Ontario, Canada N2L 3G1

E-mail address: ipgoulden@math.uwaterloo.ca

Department of Combinatorics and Optimization, University of Waterloo, Waterloo, Ontario, CANADA N2L 3G1

E-mail address: arattan@math.mit.ca

Current address: Department of Applied Mathematics, Massachusetts Institute of Technology, Cambridge, Massachusetts 02139 